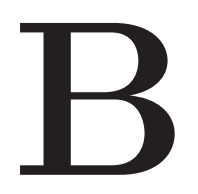

\title{
Introduction to Quantum Effects
}

W

HAT are quantum effects, how does one build an intuitive sense of them, and what do quantum effects mean? The roots of these important questions are found in wave mechanics. The previous appendix began the exploration of the quantum world with a review of quantum sizes, measurement, and the properties of light. This appendix builds on that knowledge by summarizing the history and debates of wave mechanics, which was developed at the start of the twentieth century. The appendix then introduces three quantum effects that flow from wave mechanics: uncertainty, entanglement, and superposition. These three quantum effects form the basis of the quantum computing, communication, and sensing technologies discussed later in this work.

\section{B.1 Wave Mechanics}

What are quantum effects and what do they mean? Consider Richard Feynman ${ }^{1}$ (pronounced Fine-man), the American physicist who was also a great popularizer of science. Feynman was critical of attempts to understand the meaning of quantum mechanics. As he made clear in numerous public speeches and lectures, quantum mechanics is a set of mathematical equations that explain experiments and observed

\footnotetext{
${ }^{1}$ Feynman shared the 1965 Nobel Prize in physics with Sin-Itiro Tomonaga and Julian Schwinger "for their fundamental work in quantum electrodynamics with deep-ploughing consequences for the physics of elementary particles." NobelPrize.org, "The Nobel Prize in Physics 1965" (2019).
} 
phenomena. "I think I can safely say that nobody understands quantum mechanics," was one of his more memorable quotations.

Yet, despite these recommendations, physicists and the public alike are thirsty for some kind of intuitive understanding of what these quantum equations mean. Such an understanding is especially important for this book, since our goal is to provide insight into quantum information science and its implications without delving into the underlying physics and math. The remainder of this section, describes four critical observations that are the basis of quantum physics and are critical for grasping what is special and different about quantum technologies.

\section{B.1.1 Quantum Swirls}

What happens at the quantum domain doesn't stay in the quantum domain: quantum effects are visible all around us if one knows where and how to look. Perhaps the most obvious evidence is what physicists in the early twentieth century called the wave-particle duality. This duality indicates that the physical building blocks of reality mass and energy - result in effects at the macro-scale that are reminiscent of both waves and particles. This confounded physicists for a time, as they assumed things like light and matter had to be $e i$ ther discrete particles or waves oscillating in some kind of medium. The birth of quantum physics resulted from the realization (and the corresponding mathematics) that light and matter are neither waves nor particles, that there is no medium, and that tiny microscopic objects don't behave like tennis balls.

The swirl of colors in a soap bubble (Figure B.1) illustrates a quantum process at work. The colors are created by interference between two wave fronts: the light reflecting off the front side and the back side of the soap film. This demonstrates the wave-like properties of light. Different colors are caused by light with different wavelengths, unquestionably demonstrating that light is a wave. Such wave-like behavior is not limited to light: similar effects can be observed in tiny "particles" of matter (such as electrons), and even in large organic molecules. ${ }^{2}$

On the other hand, if you take light from the Sun and shine it on a piece of metal, you'll discover that the Sun's ultraviolet light - the same kind of invisible light responsible for sunburn - can dislodge

${ }^{2}$ Gerlich et al., "Quantum Interference of Large Organic Molecules" (2011). 


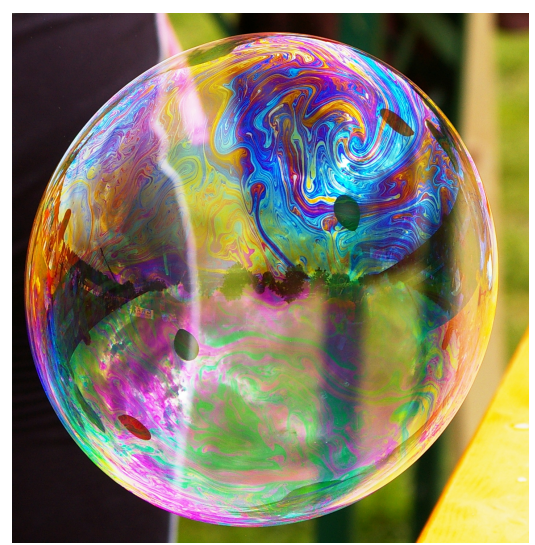

Figure B.1. The colorful swirls in a soap bubble are the result of constructive and destructive interference of light reflecting against the inside and outside soap film walls. The changing distance between the two walls at different points in the bubble simultaneously results in constructive interference of some colors and destructive interference of others. As a result, the soap film seems to possess different colors at different points. Image CC-BY-SA Wikimedia user Werner100359.

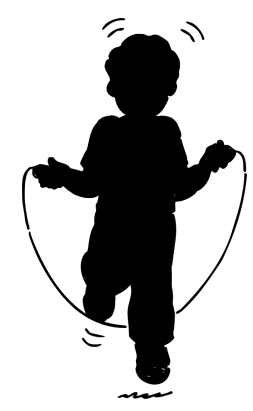

Figure B.2. In this illustration of a young child jumping rope, the movement of the rope describes a circular wave. The rope is the wave's medium, the rope's wavelength twice the child's arm-span, and the frequency is the number of times per second that the rope passes under the child's legs. The wave's amplitude is distance from the line between the child's hands and the rope's midpoint at the child's ankle.

electrons from the surface of the metal, producing a slight voltage, while light from the red end of the spectrum can't. This is called the photoelectric effect.

What is odd about the photoelectric effect, though, is that whether or not light produces electricity when it hits the metal depends entirely on the light's color - its wavelength or frequency - and not the light's brightness or intensity.

There are two numbers that describe a wave propagating through a medium: the wave's amplitude and its frequency. The amplitude is how much the wave displaces the medium from its resting state, also called its ground state. The frequency is how many times per second the wave causes the medium to oscillate. (See Figure B.2.)

Classical physics says that the energy transferred by a wave is proportional to its amplitude. If light were a wave, its brightness 


\section{On Quantum and Elementary Particles}

In this section we've used the imprecise phrase quantum particles to describe very small particles, whereas most texts would probably have used the term elementary particles, meaning the smallest particles that are the building blocks of matter.

Electrons, protons, and neutrons were once called elementary particles because they were thought to be the fundamental building blocks of matter. Today, most physicists subscribe to the Standard Model which describes the hundred-or-so subatomic particles out of which the universe is thought to be made. Under the Standard Model, the term elementary particle is reserved for leptons and quarks. Electrons are leptons, whereas protons and neutrons are made up of quarks. Protons in particular are made up of two Up and one Down quarks, while the neutron is made up of two Down and one Up quarks. Quarks and leptons are both called fermions. There are 24 kinds of fermions: the six quarks (named up, down, strange, charm, bottom, and top), six leptons (the electron, electron neutrino, muon, muon neutrino, tau particle, and tau neutrino), and, for each lepton, its antiparticle.

The photon is neither lepton nor quark: it is a boson, which is the name used for particles that follow Bose-Einstein statistics. The key difference between fermions and bosons is that fermions obey the Pauli exclusion principle, which means that two fermions cannot be in the same place and in the same state at the same time, while any number of bosons can be packed together. Light is a boson (and in particular, a gauge boson), which is why many photons can be packed together in a laser. Likewise helium is a boson (it's actually called a composite boson), which allows it to form a superfluid when it is cooled close to absolute zero. 


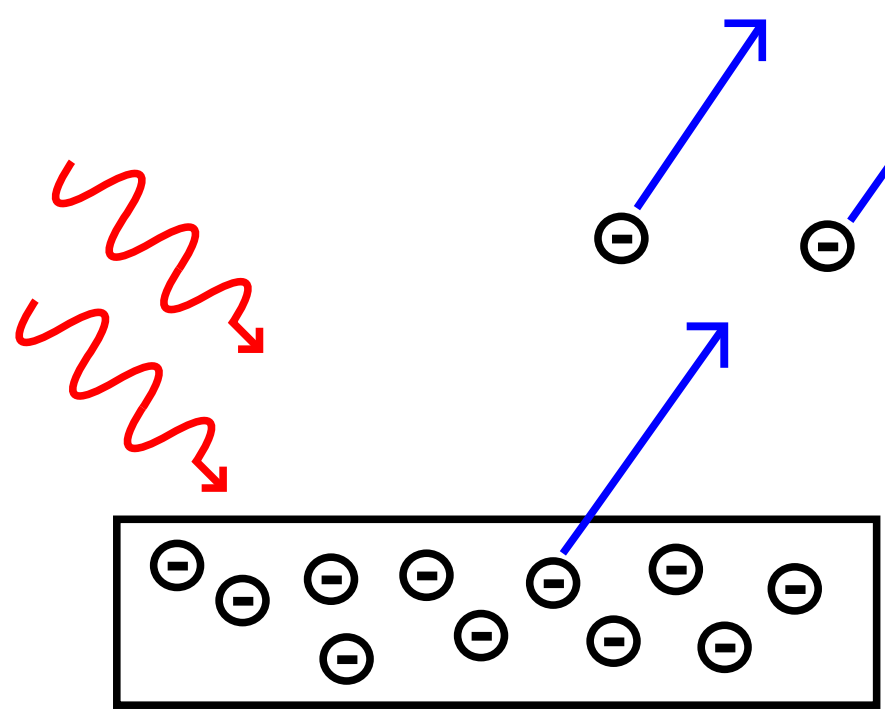

Figure B.3. The photoelectric effect results when light striking metal causes electrons to eject. Einstein explained the effect by saying that the energy of light was carried in individual particles, which are now called photons, and that the energy of those particles is proportional to the frequency of the light, with photons from higherfrequency light having more energy. Image CC-BY-SA Wikimedia user Wolfmankurd. 
would be the wave's amplitude, and its color would be its frequency. Einstein's explanation required that light be viewed as a stream of particles, not waves, with the energy of each particle depending, perplexingly, on that particle's "wavelength."

\section{B.1.2 Light: Newton Thought It Was a Particle}

The nature of light was a centuries-long riddle for scientists. Just what is light, and how does it work? Why are some things different colors? Why is there color during the day but not at night? Teasing out which of the observed effects were due to the underlying nature of light, and which were due to the nature of the observer, took literally thousands of years of scientific work.

In 1704, Sir Isaac Newton published his treatise Opticks, in which he showed how the fundamental nature of light could be revealed through the use of prisms and mirrors. In that book Newton also promoted what was called the corpuscular theory of light, the idea that light was made up of tiny particles called corpuscles, a concept first proposed by Descartes in 1637. Newton's work on light bolstered the corpuscular theory, since light travels in straight lines and reflects from mirrors at right angles, like billiards bouncing off a pool table's bumpers. Waves traveling across the surface of a bath tub or lake just don't act that way. Furthermore, Newton argued that if light were a wave, then one would see interference fringes in the boundary between light and darkness that arise when an object with a sharp edge casts a shadow.

For all of Newton's prestige, the corpuscle theory really didn't do a good job explaining why light has color. But the real nail in the theory's coffin was the discovery that light in fact did produce interference patterns.

\section{B.1.3 Light: It Acts Like a Wave}

By the end of the eighteenth century, physicists had a basic understanding of waves from observing their behavior in water. For example, physicists understood that waves traveled through some kind of medium, causing it to cycle up and down.

Recall from the illustration of the child jumping rope, the height of a water wave is its amplitude, while the distance between the peaks is the wavelength. The frequency is the number of times per second that the rope passes over the child's head. The frequency and the wavelength of a wave are inversely related. 
Interference happens between waves when two (or more) waves meet and pass through each other: where the wave peaks align, the interference is constructive: the peaks add together, increasing their intensity. Where a peak aligns with a trough, the interference is destructive, and the waves cancel each other out. You can readily perceive this effect with sound by having a colleague stand a few feet away from you with two tuning forks. If your colleague strikes both forks and holds them a foot apart, you will perceive the sound to be louder and quieter as you approach or retreat from your friend's position. The change in volume is caused alternately by constructive and destructive inteference of the sound waves, which are now known as compression waves in the medium of air. ${ }^{3}$

In 1801, the British scientist Thomas Young devised an experiment that established beyond a doubt that light has wave-like properties. In the experiment (see Figures B.6 and B.7), a stream of light travels through two slits in a black plate. Young reasoned that if light were made out of tiny ball-like particles (Newton's "corpuscles"), the particles passing through each slit would produce a slightly larger rectangular line on the screen. And indeed, that's what happens if the slits are large. But when the slits are small, an interference pattern emerges, showing that light has wave-like properties.

At the time, Young and others assumed this meant that light was actually a kind of wave, like sound, and not a kind of particle as Newton had hypothesized. (Full-length books have been devoted to the two-split experiment ${ }^{4}$ the complexity of which will not be fully conveyed here.)

Of course, once you know what to look for, interference shows up in all kinds of places: put a lightly curved watch glass on a piece of white paper and illuminate it from above, and you will observe a bull's-eye pattern of rainbows (if illuminated with white light), or light and dark circles (if illuminated with monochromatic light). These circles are called Newton's rings (Figure B.4) and they are an interference fringe; they allow you to make precise measurements

\footnotetext{
${ }^{3}$ The invention of the vacuum pump in 1650 by Otto von Guericke and the discovery of air pressure was a major driver of the scientific and engineering revolutions that were to follow. For an excellent history of vacuum science, see Grant, Much Ado About Nothing: Theories of Space and Vacuum From The Middle Ages to The Scientific Revolution (2008).

${ }^{4}$ Ananthaswamy, Through Two Doors at Once: The Elegant Experiment That Captures The Enigma of Our Quantum Reality (2018).
} 


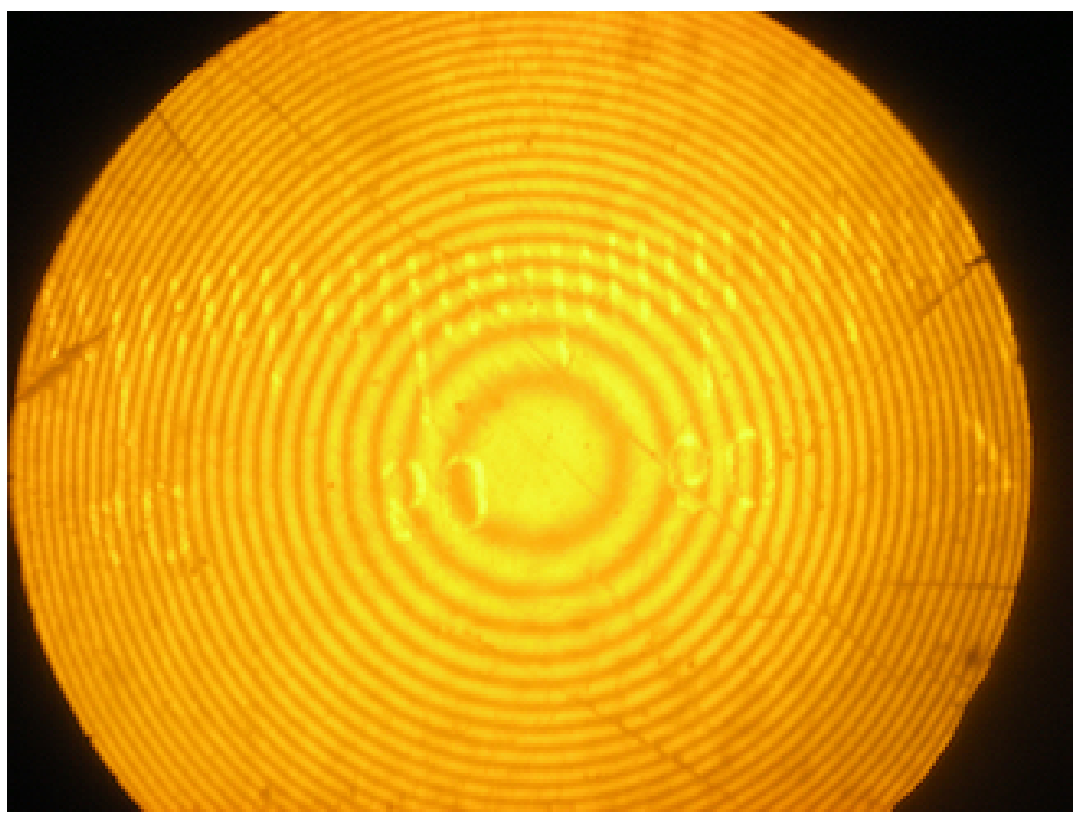

Figure B.4. Newton's rings observed through a microscope. The pattern is created with a $20 \mathrm{~cm}$ convex lens illuminated from below by a monochromatic sodium lamp. The scale has $100 \mu \mathrm{m}$ increments. Image CC-0 by Wikicommons user Warrencarpani.

regarding changes in distance or pressure between the glass and the paper.

Physicists have repeatedly made good use of light's wave-like properties since 1801 - and they continue to do so to this day.

Consider the use of the Doppler Effect, which is the term that physicists use to describe the apparent upwards shift in frequency when the distance between a wave emitter and an observer is decreasing, and the corresponding apparent decrease in frequency when that distance is increasing. If an emergency vehicle with a blaring siren approaches and then speeds past you on a street, the siren's wail will be heard at a higher pitch as the vehicle approaches and passes a listener, and then at a lower pitch as the vehicle recedes. This change in pitch was first characterized by the Austrian physicist Christian Doppler in 1842. The shift is caused because the decreasing distance between the vehicle and the listener effectively results in the peaks of each sound wave hitting the listener's eardrum faster than they would if there was no relative motion between the two. Likewise, when the vehicle is receding, the sound waves are effectively stretched out. 


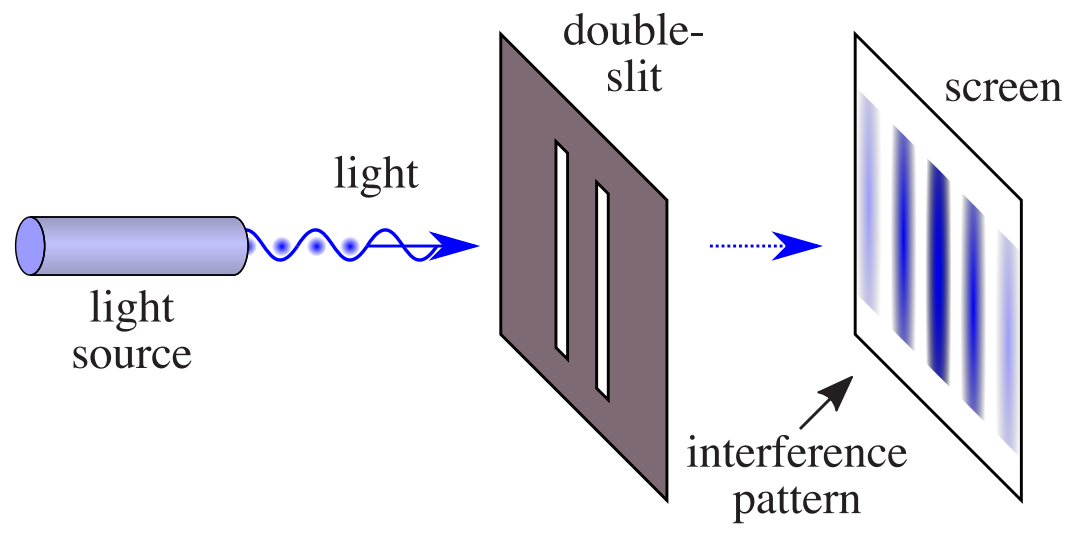

Figure B.5. Thomas Young's dual-slit experiment demonstrated that light has wavelike properties. In the double-slit experiment, light from an emitter travels through two slits and forms an interference pattern on the screen, just as waves passing through two holes in a water break cause interference on a lake. Image CC-BY-SA by NekoJaNekoJa with author edits.

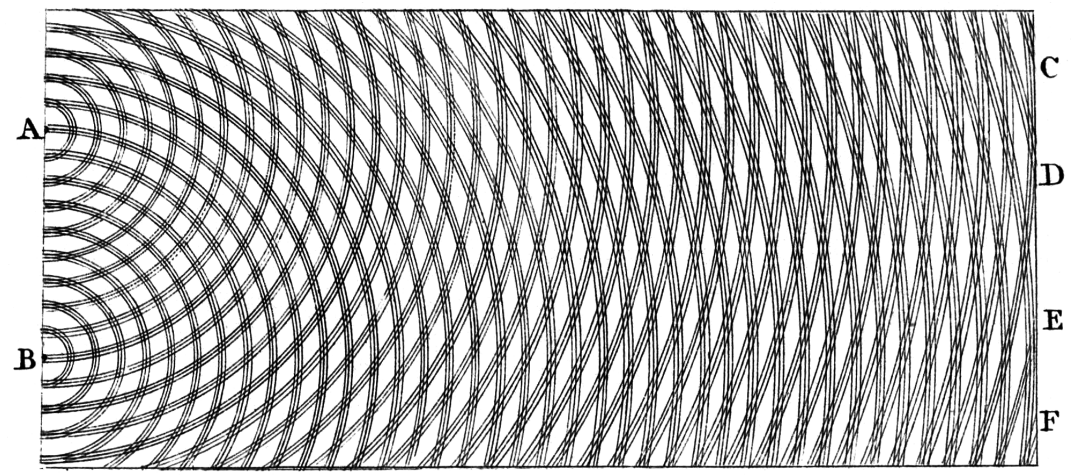

Figure B.6. This is a drawing from Thomas Young's notebook showing how light rays traveling from two point sources $A$ and $B$ result in constructive and destructive interference. In the dual-slit experiment, each slit can be thought of as a stack of point sources that emit light solely in the horizontal plane. Image public domain. 
In 1929, US astronomer Edwin Powell Hubble showed that the light from stars and distant galaxies is also shifted red, implying that every galaxy in the night sky is moving away from us. This was the first evidence that the universe is expanding, which is indirect evidence for the Big Bang theory. Red shift measurement depends on the fact that light has wave-like properties, and the fact that light's behavior at macroscopic scales almost perfectly approximates the behavior of a wave moving through some kind of medium. (Indeed, in 1873, James Clerk Maxwell published his theory of electromagnetism with his now-famous set of equations that beautifully described the behavior of light, basing that description on the core idea that light was a wave.)

Physicists can also use the wave-like way that light casts interference patterns to make precise measurements of distance using a technique known as interferometry. The technique works by splitting coherent, monochromatic light from a single source into two beams which reflect off two different mirrors and are then recombined: if the distances are exactly the same, then the peaks from one path precisely match the peaks from the other, the interference is positive, and the resulting light is the same brightness as the original source before splitting. But if one path extends precisely one-half of a wavelength longer, then the peaks from one path line up with the troughs from the other, and the interference is destructive: the two beams cancel each other out.

In 2015, a pair of $2000 \mathrm{~m}$-long L-shaped interferometers at the Laser Interferometer Gravitational-Wave Observatory (LIGO) were able to detect minute distortions in the curvature of space-time resulting from gravity waves generated by the collision of two neutron stars in galaxy NGC 4993, 144 million light-years from Earth. The collision was also detected by Virgo interferometer operated by the European Gravitational Observatory, with scientists at both labs winning the 2017 Nobel Prize in physics as a result. As an aside, not only did the experiment demonstrate the existence of gravitational waves, it also showed that they travel at the speed of light, as predicted by Einstein's general theory of relativity.

\section{B.1.4 Light: How Can It Possibly Be a Wave?}

It turns out that light can't be a wave for two very basic reasons: there's no medium to vibrate, and light comes in discrete, countable units - something that waves just don't do. 
Waves on the surface of a lake result from bulges and troughs in water, while sound waves result from compression of air. Water and air are both a medium that transmits wave energy. Given that light was obviously a wave phenomenon, scientists of the nineteenth century ${ }^{5}$ wanted to better understand the medium that light was moving through. They called that medium the Luminiferous aether, or simply aether (or even ether).

Physicists knew that light could travel through air, water, glass and even a vacuum, so aether had to be everywhere, penetrating everything. And since the Earth was rotating, light traveling in the direction of the Earth's motion should be impacted by the aether differently than light traveling at right angles. This created an opportunity for measurement. In 1887, Albert A. Michelson of the Case School of Applied Science and Edward W. Morley of Western Reserve University, both in Columbus, Ohio, built a massive interferometer in the basement of a university dormitory to measure this effect of the earth's movement through the aether. ${ }^{6}$ They failed to find any effect, demonstrating that there was no aether for light to be traveling through.

Another problem with the wave theory of light is that it can't mathematically explain the amount of light emitted by objects when they heat up. If you have ever worked with a furnace, or even an electric stove, you know that when things like metal rods get hot, they tend to glow - first red, then orange, and eventually bright white. This is called black-body radiation, because the color of the light is independent of the color of the object being heated - it even comes off objects that are pure black.

In the late-nineteenth century, physicists started measuring the light coming off of hot objects and then trying to develop mathematical models to explain their measurements. Based on the wave theory of light, the amount of ultraviolet radiation coming off a hot piece of metal should have been significantly higher than the amount of blue or green light - but it was significantly less. In fact, predictions based on Maxwell's equations indicated that as the frequency of light steadily increased, the amount of light coming off should steadily in-

\footnotetext{
${ }^{5}$ Although much of the work to detect the aether took place in the nineteenth century, theories regarding the aether date back to Robert Boyle and Christiaan Hyugen Huygen, Traité de la Lumière (1690) in the seventeenth century.

${ }^{6}$ The Case School of Applied Science and Western Reserve University merged in 1967 to form Case Western Reserve.
} 
crease as well, such that an infinite amount of light was coming off with light that had an infinite frequency. Clearly that wasn't happening. This mismatch between theory and observation was called the ultraviolet catastrophe.

In 1900, the German physicist Max Planck published a mathematical theory that properly predicted radiation emitted by black bodies. The theory assumes that the light emitted by black-body radiation is quantized at specific levels. Planck didn't go so far as to say that quantization was inherent in all kinds of light. Still, this work earned Planck the 1918 Nobel Prize in Physics, "in recognition of the services he rendered to the advancement of Physics by his discovery of energy quanta."

Five years later, Einstein built upon Planck's work and suggested that light itself was quantized, and not merely the energy levels at which light is radiated from black bodies. With this leap of intuition, Einstein was able to explain the aforementioned photoelectric effect. Einstein's 1905 explanation of how it works ${ }^{7}$ was experimentally confirmed by Robert Millikan in 1915 at the University of Chicago. ${ }^{8}$ It was for this work that Einstein was awarded the 1921 Nobel Prize in Physics "for his services to Theoretical Physics, and especially for his discovery of the law of the photoelectric effect."

Thus, the inescapable conclusion of more than a century's worth of physics research is that light is both a wave and a particle - or, more accurately, that physicists can construct experiments in which light has observable effects that appear similar to the wave-like effects that physicists can observe in sound waves, and the particle-like properties that physicists can observe in objects like tennis balls.

Before the invention of quantum mechanics, some physicists called this the "wave-particle duality," a name that has unfortunately persisted to this day (although the authors will try not to use that phrase elsewhere in this book). Einstein explained it this way in 1938:

It seems as though we must use sometimes the one theory and sometimes the other, while at times we may use either. We are faced with a new kind of difficulty. We have two contradictory pictures of reality; separately nei-

\footnotetext{
${ }^{7}$ Einstein, "Über Einen Die Erzeugung Und Verwandlung Des Lichtes Betreffenden Heuristischen Gesichtspunkt (On The Production and Transformation of Light From a Heuristic Viewpoint)" (1905).

${ }^{8}$ American Physical Society, "Robert A. Millikan" (n.d.).
} 
ther of them fully explains the phenomena of light, but together they do. ${ }^{9}$

In fact, as far as light goes, quantum theory explains virtually all observations that humans have ever made. The one exception is that quantum theory does not explain the curvature of space-time, which clearly affects the way that light bends around massive gravitational objects like stars and black holes. But with the exception of gravity, the quantum theory of light appears to be complete.

The word photon itself was coined by Gilbert N. Lewis in a 1926 letter to Nature. ${ }^{10}$

It's Not Just Light: Everything Has Both Wave-like and Particle-like Properties

This apparent combination of both wave-like and particle-like effects is not confined to light: all matter has wave-like properties, from tiny particles of matter like electrons, to much larger molecules, to planets and stars. More to the point, these waves can even be measured - at least in the case of electrons and molecules.

In 1924, Louis-Victor de Broglie derived an equation that relates the wavelength of any object $(\lambda)$ to momentum ${ }^{11}(p)$ and Planck's constant $(h)$. That equation is:

$$
\lambda=\frac{h}{p}
$$

de Broglie's equation implied that everything has a measurable wavelength (or, if you prefer, a measurable frequency). When scientists went out to measure these waves, they found them ... with precisely the wavelength that de Broglie's equation predicts. The first confirmation came from Bell Labs in 1927, ${ }^{12}$ when slow-moving electrons hitting crystalline nickel were shown to refract (at the quantum level, the arrangement of atoms in crystalline nickel looks like a lot of ridges or slits). The idea that matter has wave-like properties was so radical, and the confirmation was precise, that the Nobel committee awarded de Broglie the 1929 Prize in Physics "for his discovery of the wave nature of electrons."

\footnotetext{
${ }^{9}$ Einstein and Infeld, The Evolution of Physics: The Growth of Ideas From Early Concepts to Relativity and Quanta (1938).

${ }^{10}$ Lewis, "The Conservation of Photons" (1926).

${ }^{11}$ Recall that the momentum of an object is its mass times its speed.

${ }^{12}$ Davisson and Germer, "Reflection of Electrons by a Crystal of Nickel" (1928).
} 


\section{$\mathrm{S}_{2}^{\prime} \cdot$}

$\mathrm{S}_{1}^{\prime} \bullet$
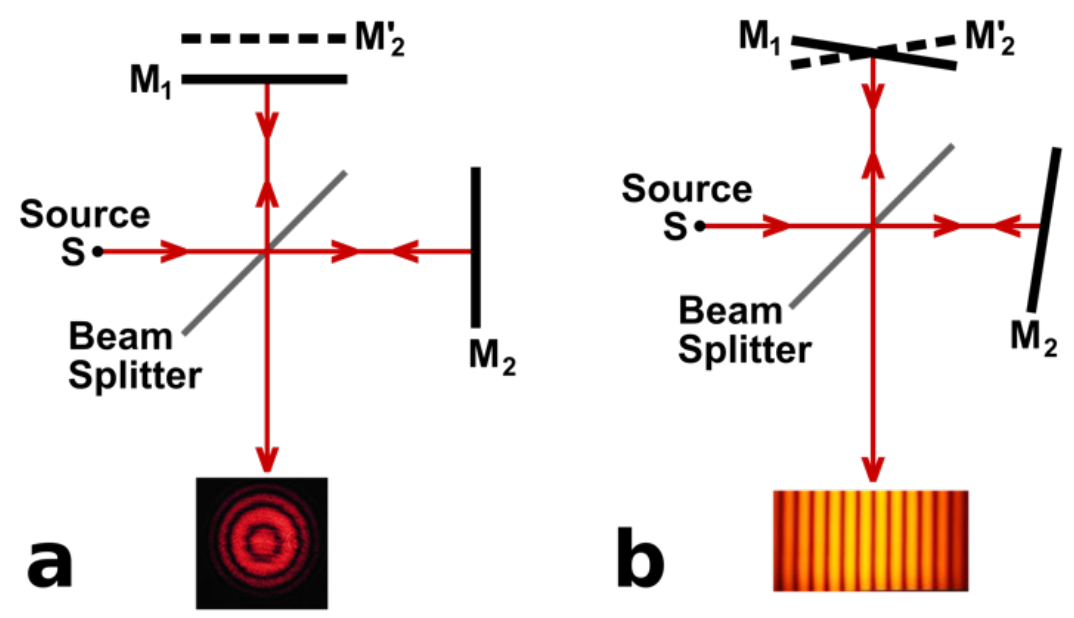

Figure B.7. A Michelson interferometer uses a source of light and a beam splitter to make precise measurements of the relative angles and distances of the two mirrors $M_{1}$ and $\mathrm{M}_{2}$. The bull's-eye pattern results from rings of constructive and destructive interference between the convex lens and a plane of glass, with each band representing an increase in separation between the planes of glass equal to an additional wavelength of light. If light were actually a wave traveling through the aether, then the interference pattern would be smeared when the direction of the earth's movement when the movement was aligned with an axis of the lens; the resulting pattern would be a series of bars, rather than a bull's-eye. Michelson and Morley never observed such a pattern: this was taken as proof that the Earth is not moving through an aether medium. Image CC-BY Wikimedia user Stigmatella_aurantiaca. 
In 1999, researchers at the University of Vienna demonstrated diffraction patterns from $\mathrm{C}_{60}$ "buckyballs" (fullerenes), which have a diameter of roughly $0.7 \mathrm{~nm}$, meaning that even large molecules have observable wave-like properties. Larger objects, like books, cars, and people, have wavelengths, although they are tiny, even by quantum standards - that's because an object's wavelength is inversely proportional to its mass. In the case of a $58 \mathrm{~g}$ regulation tennis ball being served at the $263 \mathrm{~km} / \mathrm{h},{ }^{13}$ the fastest serve on record, $p$ is $0.058 \mathrm{~kg}$ times $4383 \mathrm{~m} / \mathrm{s}$, giving a wavelength of $2.6 \times 10^{-37} \mathrm{~m}$, which is 22 orders of magnitude smaller than the diameter of a proton. That's the reason why the wave-like nature of particles typically isn't visible in our day-to-day classical world: the wavelengths are so small that they can be easily ignored.

In 2018, scientists at Hitachi demonstrated a version of the dualslit experiment using an advanced device that can detect individual electrons and show them on a screen. ${ }^{14}$ In the experiment, individual electrons are accelerated in a vacuum to 50000 volts, which is $40 \%$ the speed of light. The electrons then pass on either side of an electron biprism (a very thin wire with a negative charge) and then smash into a detector. The team posted a video on YouTube showing the screen as each additional electron arrives. Since just 10 electrons travel through the device each second, there is no way for the electrons to interfere with one another - most of the time, there is no electron in the device. And indeed, as the first few electrons appear on the screen, they appear to be randomly placed. But after the experiment runs for 20 minutes, a clear pattern of bright and dark bars appears on the screen. This is the characteristic interference pattern of the dual-slit experiment.

So what's going on?

The Hitachi experiment shows that the electrons are arriving at the detector in accordance with a probability distribution. The bright bars are where electrons have a high probability of appearing; the dark bars are where the probability is low. By measuring the distances, it's possible to calculate the wavelength that would cause such a pattern to appear: it is the same wavelength that is revealed by the de Broglie equation.

13 en.wikipedia.org/wiki/Fastest_recorded_tennis_serves

${ }^{14}$ www.hitachi.com/rd/portal/highlight/quantum/doubleslit/index.html 
Paradoxically, the only way to make sense of this experiment is to let go of the classical notions that electrons are little particles that travel along paths predetermined by the mass, charge and momentum. Instead, think of the electron gun, the two slits, and the detector as a single system. The release of each electron, its acceleration to 50000 volts, its travel through the slits, and its detection are not four distinct events, but a single action that takes place in space and time, transferring a tiny bit of mass from the electron emitter and a tiny bit of energy from the acceleration plates to the detector. This transfer of mass and energy can be described by a single equation that, when solved, provides the probability that is different for different points of the single electron detector.

If you crave a classical explanation for what is happening, consider a gambler who is rolling a pair of dice. With each role, there is a chance that the sum of the two dice will be 2 , or 12 , or any number in between. There's no way for the gambler to predict the next roll of the dice, but the gambler knows that, over time, a roll of 7 is the most likely. Likewise, in the Hitachi experiment, there's no way to predict the location of each electron, but over time the pattern of light and dark bars will clearly emerge.

Light and Matter: whatever it is, it's described by Schrödinger's wave equation and Heisenberg's matrices.

In 1925, the 24-year-old Werner Heisenberg was working as an assistant to Max Born at the Institute of Theoretical Physics at the University of Göttingen. There Heisenberg developed a mathematical formulation based on matrix math that accurately described the interactions between light and matter that scientists had been able to precisely measure up to that point. ${ }^{15}$ The following year, Erwin Schrödinger developed what is now called the Schrödinger Wave Equation which does the same thing, but which is based on partial differential equations. The two formulations are in fact mathematically equivalent, although it is sometimes easier to use one formulation, and sometimes easier to work with the other. It is these systems of equations that are called quantum mechanics.

${ }^{15}$ While the phrase "the interaction of light and matter" may sound quite grandiose, most of these interactions are simply what happens when electrons in atoms absorb a photon and jump to a higher energy level, and when electrons drop back down to a lower energy level, emitting a photon. 
For many people, philosophers and physicists alike, the challenge of quantum mechanics comes when trying to ascribe meaning or an "interpretation" to these equations. Our recommendation here is the same as Richard Feynman's: don't try to ascribe sense or meaning to the equations, just accept that they accurately predict experimental observations. Leave it at that.

For example, if you set up the equation to describe the position of an electron around a hydrogen atom, you can then take the value of function at any point in 3-dimensional space, square the value of the function and then take the absolute value (that is, if the number is negative, make it positive), and the result predicts the density of the electron cloud at that point over the course of many observations of many different atoms. This is called the Born rule, named after Max Born, who suggested the relationship in 1926. Viewing $\left|\Psi^{2}\right|$ as a probability is clean mathematically, but it raises many problems philosophically.

The first problem has to do with the formulation of squaring the number and then taking the absolute value. One has to do this because the function itself is a complex, vector function. That is, at any point $(\mathrm{x}, \mathrm{y}, \mathrm{z})$ the function evaluates to a number with two components, one that is a real number (such as 0.5 or -0.2 ) and one that is a complex number (such as $0.25 i$ ). Recall that $i$ is the number that, when multiplied by itself, produces -1 . That is, $i^{2}=-1$ or $i=\sqrt{-1}$. This is why it is necessary to both square the wave equation and to take its absolute value: because probabilities have to be positive. (More exactly, the value of the function is actually multiplied by its complex conjugate.)

So what does the wave equation actually mean? It turns out that we do not really know. The Born rule produces the right answers, but we do not know why. Specifically, we do not know why the rule works, and we do not know what it means philosophically about the nature of reality. This is what Feynman meant when he said "I think I can safely say that nobody understands quantum mechanics." Feynman was making a point about epistemology.

Put another way, the wave equation accurately describes quantum phenomena observed in experiments. But from an epistemological viewpoint, no one has any first-hand knowledge what these equations actually mean. Only one of our senses can perceive quantum events directly - specifically, the dark-adapted human eye can perceive individual photons. But that's about it. When it comes to 
electrons, protons, atoms, or even molecules, our senses are limited to indirect measurements. When it comes to air pressure, we do not perceive air molecules pounding against our skin as the result of Brownian Motion. In fact, we do not perceive air pressure at all, which is why its existence was unknown for most of human history.

This kind of empirical relativism is fundamentally unsatisfying to many, and as a result there have been many efforts to interpret the meaning of the wave equation into words that make sense to humans. There is also an ongoing effort in theoretical physics called Quantum Reconstruction that seeks to derive the Born rule, as well as other seemingly arbitrary aspects of quantum mechanics, from a significantly smaller set of fundamental postulates. Physicist John Wheeler advocated this approach in 1983, arguing that there should be laws of physics that emerge from mathematics, what he called "law without law":

[A]ll of Physics in my view, will be seen someday to follow the pattern of thermodynamics and statistical mechanics, of regularity based on chaos, of "law without law." Specially, I believe that everything is built higgledy-piggledy on the unpredictable outcomes of billions upon billions of elementary quantum phenomena, and that the laws and initial conditions of physics arise out of this chaos by the action of a regulating principle, the discovery and proper formulation of which is the number one task. ${ }^{16}$

For many people, this is ultimately what is most unsettling about quantum mechanics: in practically every other field of science and social science, scientists base their theories on clear, consistent mental models. They perform mental experiments to see how those models work. They then put math to the models, and finally, collect data to see if observed phenomena agree with the models. That's the basic process started a thousand years ago in ancient Arabia, when the scientist Ibn al-Haytham conducted experiments in optics and used the results of his experiments to prove one theory of vision and disprove another. ${ }^{17}$ It's the approach that Newton used to create his laws of motion, it's the basic process of economics.

${ }^{16}$ Wheeler, "On Recognizing 'Law Without Law,' Oersted Medal Response at The Joint APS-AAPT Meeting, New York, 25 January 1983” (1983).

${ }^{17}$ al-Haytham, Book of Optics (1011). 
However, this approach is different from the approach the Pythagoreans used to invent mathematics, that Aristotle used to explain the world, and that Einstein used to create his theory of relativity. In those cases, people sought to create an intellectual framework that was internally consistent. Indeed, when Einstein's assistant Rosenthal-Schneider asked him what he would have done if the 1919 transit of Mercury across the Sun did not confirm the General Theory of Relativity, Einstein replied, "Then I would feel sorry for the good Lord. The theory is correct." 18

\section{B.2 Quantum Effects 1: Uncertainty}

In early 1926, Heisenberg was invited to give a talk on the matrix mechanics in Berlin. In the audience were Max Planck (who won the 1918 Nobel Prize in Physics "in recognition of the services he rendered to the advancement of Physics by his discovery of energy quanta"), Max Theodor Felix von Laue (who won the 1914 Nobel Prize in Physics "for his discovery of the diffraction of X-rays by crystals"), Walther Hermann Nernst (who discovered the third law of thermodynamics and had won the 1920 Nobel Prize in Chemistry "in recognition of his work in thermochemistry"), and Albert Einstein (who as previously noted had won the 1921 Nobel Prize in Physics for the photoelectric effect).

This assemblage of some of the world's foremost physicists must have been quite intimidating to the 25-year-old Heisenberg! He could probably not have imagined at the time, but in just six years he would earn the 1932 Nobel Prize in Physics, "for the creation of quantum mechanics, the application of which has, inter alia, led to the discovery of the allotropic forms of hydrogen."

Einstein invited Heisenberg to come back to his house after the lecture, and the two discussed the fundamental relationship between theory and experimental observation. ${ }^{19}$ According to Heisenberg, ${ }^{20}$ Einstein argued that a physicist must start with a theory, and from that decide what observations are possible (and presumably which experiments to perform). Heisenberg, in contrast, said that one must start with what is observed during the course of an experiment. If

\footnotetext{
${ }^{18}$ Batten, "Subtle Are Einstein's Thoughts" (2005).

${ }^{19}$ This was the first time that Heisenberg was to meet Einstein, but not the last: the two had a lifelong relationship which Heisenberg wrote about in his posthumously published book, Encounters with Einstein.

${ }^{20}$ Heisenberg, Encounters with Einstein (1983).
} 
nothing can be observed, then, from the point of view of physics, there is nothing to explain.

This difference in opinion between Einstein and Heisenberg proved to be foundational, influencing how the two would view physics for decades to come.

It's important to realize that the word observation here has two meanings, one very specific, the other quite general. The specific meaning is quite literally something that a person (presumably a physicist) can observe, or more accurately, perceive. An observation might be a flash of light, the sound of an explosion, or even the movement of a dial. The second meaning of observation is more general: since scientific instruments have lights and dials, the word observation really means anything that can be measured scientifically. And since sensitive scientific instruments can detect a single electron or photon, this really means anything that can interact with an atom or an atomic particle in some detectable manner. If something cannot be detected, then there is no reason to explain it with a theory indeed, it is not possible to explain with a theory, because there is (by definition) no way to prove if the theory is right or wrong.

Heisenberg returned to Copenhagen and continued to develop quantum mechanics, where he discovered another curious aspect of the theory: according to his math, it should not be possible to precisely determine the position and the speed of an object simultaneously. This was not a consequence of poor instrumentation, it was a result of the underlying physics. This is because the act of measuring something requires interacting with that thing. For example, if you wish to measure the size of a coin, you can put the coin against a ruler, but then you need to bounce light off the coin and into your eye so that you can observe the coin's dimensions. And each time a photon bounces off the coin, there is a physical consequence. Heisenberg called this the indeterminacy principle; today it is commonly called the Heisenberg Uncertainty Principle.

To understand the uncertainty principle, let's follow Heisenberg's thought processes. Let's say that one wants to describe the quantum state of a silver coin. To start, one would need to note the precise position of every silver atom that the coin contains. To do this, one could use a microscope that bounced light off each atom on the atom's surface to carefully establish each atom's position. One could capture this bounced light and slowly measure the state of the entire object. 


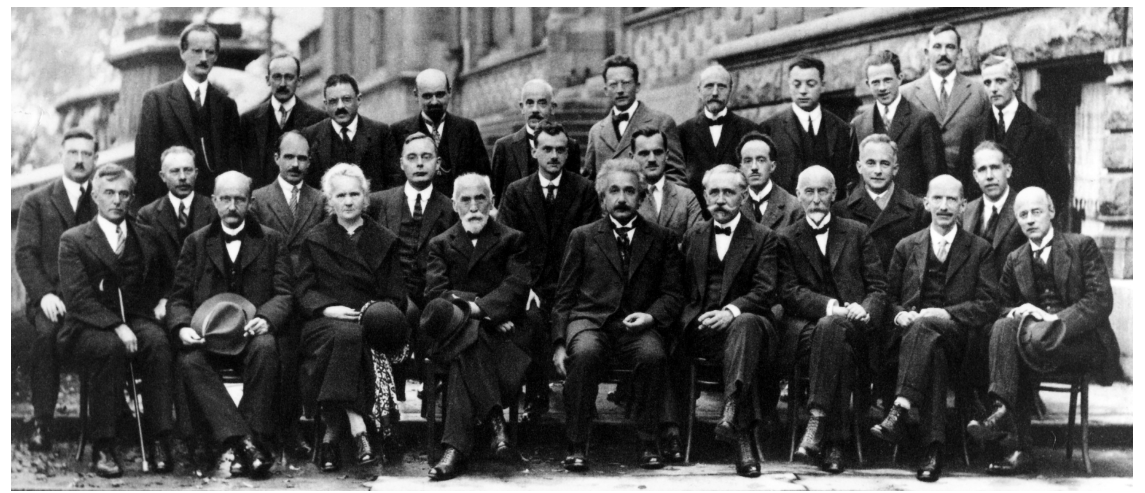

rear:

A. Piccard

Th. de Donder

E. Henriot

P. Ehrenfest

E. Herzen

W. Heisenberg

E. Schrödinger

R. H. Fowler

J. E. Verschaffelt W. Pauli

middle:

P. Debye

M. Knudsen

L. Brillouin

P. A. M. Dirac

A. H. Compton

W.L. Bragg

N. Bohr

L. de Broglie

H. A. Kramers

M. Born

Front:
I. Langmuir
M. Planck
M. Curie
H.A . Lorentz
A. Einstein
P. Langevin
Ch.-E. Guye
C. T. R. Wilson
O. W. Richardson

Figure B.8. Fifth Solvay Conference, Brussels, October 24-29, 1927. Photograph by Benjamin Couprie, Institut International de Physique Solvay.

This is the thought experiment that Heisenberg devised in 1927, although to be accurate, Heisenberg's thought experiment involved finding the location of a single electron using an optical microscope, rather than identifying all of the atoms in a coin. But the basic idea is the same.

By 1927, it was well established that light is quantized - it was six years after Einstein received his Nobel Prize, after all. So Heisenberg's microscope has to be using photons of some sort. What kind of photons should the microscope use to measure an electron?

The year 1927 also marked the fifth invitation-only conference of the International Solvay Institute for Physics and Chemistry, which is noted for its groundbreaking discussions of quantum theory. Of the conference's 29 invited attendees, 17 were or became winners of the Nobel Prize, including Niels Bohr, Albert Einstein, Marie Curie, Paul Dirac, Werner Heisenberg, and Erwin Schrodinger. The conference photo (Figure B.8) has been compared with the Bennett photo from the 1981 Physics of Computation Conference (Figure 4.9). 
Traditional light microscopes use visible light. Referring back to Table A.2, visible light has wavelengths between $380 \mathrm{~nm}$ and $740 \mathrm{~nm}$. Photons of those sizes are great for looking at things like red blood cells, which have a diameter of roughly $7 \mu \mathrm{m}(7000 \mathrm{~nm})-$ i.e. roughly 10 times the size of the wavelength of red photons. But those photons are way too big for looking at individual atoms, let alone an individual electron. Recall that nitrogen atoms so important for quantum sensing have a radius of $0.056 \mathrm{~nm}$.

A microscope works by using lenses to focus the light passing through different parts of the object to different parts of the resulting image: this is only possible because the wavelength of the light is much smaller than the size of the object under study. If you want to measure the position of individual atoms, you need to use photons with wavelengths that are roughly the same size as an atom. Looking again to Table A.1, one can see that taking pictures of atoms requires using X-rays - and that's a problem.

Since the energy of a photon is proportional to its frequency $(E=$ $\mathrm{h} f)$, the energy is inversely proportional to its wavelength $(f=c / \lambda$, so $E=h c / \lambda)$. Those photons with the atom-sized wavelength are called $X$-rays, and each one packs so much energy that it can whack an atom far, far away from the point of impact. ${ }^{21}$

Now the stage is set for Heisenberg's discovery of the uncertainty principle. It turns out that there was no way to precisely and simultaneously measure an object's position and its momentum at the atomic level: light that could precisely determine the position of an atom would result in significant energy transfer to the atom causing it to move. Light that was weak enough so that there would be no significant transfer of energy has too large a wavelength to make precise measurements. That is, as position uncertainty decreased, momentum uncertainty had to increase, and vice versa. Heisenberg crunched through the math, and arrived at his famous equation, which can be written as:

$$
\Delta x \Delta p_{x} \geq \hbar
$$

Where $\Delta x$ is the uncertainty in position in the $x$ dimension, $\Delta p_{x}$ is uncertainty in momentum in the $x$ dimension, and $\hbar$ is the value

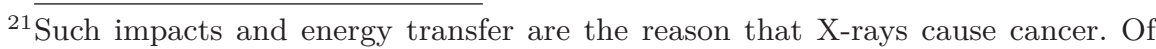
course, even ultraviolet light, with a wavelength of just $30 \mathrm{~nm}$, is still powerful enough to damage genes within cells and cause cancer: it just takes a longer cumulative exposure. 
of Planck's constant divided by $2 \pi$, known as the reduced Planck constant. It has a value of $1.05 \times 10^{-34}$ joule seconds. The joule is a measure of energy; a food calorie has roughly 4200 joules, so $\hbar$ is truly a tiny quantity by our day-to-day standards, which is the reason why we tend not to notice the inherent measurement uncertainty in the world around us.

Heisenberg's key insight - and his fundamental point of disagreement with Einstein - is that it doesn't make sense to theorize aspects of the electron, such as its position and its momentum, unless there is an actual way to measure these aspects. So it is meaningless to say that the electron has a precise position and momentum. As Heisenberg wrote:

If one wants to be clear about what is meant by "position of an object," for example of an electron...then one has to specify definite experiments by which the "position of an electron" can be measured; otherwise this term has no meaning at all. ${ }^{22}$

This kind of relationship between position and momentum is called complementarity, and there are many other instances of it in quantum physics. Perhaps the most relevant for quantum information science is the polarization of light, which turns out to be critical for quantum cryptography.

\section{B.3 Quantum Effects 2: Polarization}

Polarization is a fundamental property of light that many people are familiar with in their day-to-day experience, thanks to the widespread availability of sunglasses made from polarized filters. Polarization is also the basis of the liquid crystal displays on many computer screens and watches, which is why such displays sometimes turn black if you look at them through a pair of polarizing sunglasses.

Light polarization was discovered in 1669 by Erasmus Bartholinus (1625-1698), a Danish physicist, physician, and mathematician. ${ }^{23}$ Bartholinus noticed that when light bounces off a crystal of calcite (also known as calc-spar or Icelandic Spar), there are two reflections, as if there are two kinds of light. In fact, there are.

${ }^{22}$ Heisenberg, "Über Den Anschaulichen Inhalt Der Quantentheoretischen Kinematik Und Mechanik" (1927).

${ }^{23}$ Horváth, Polarization Patterns in Nature: Imaging Polarimetry with Atmospheric Optical and Biological Applications (2003). 
Light wave

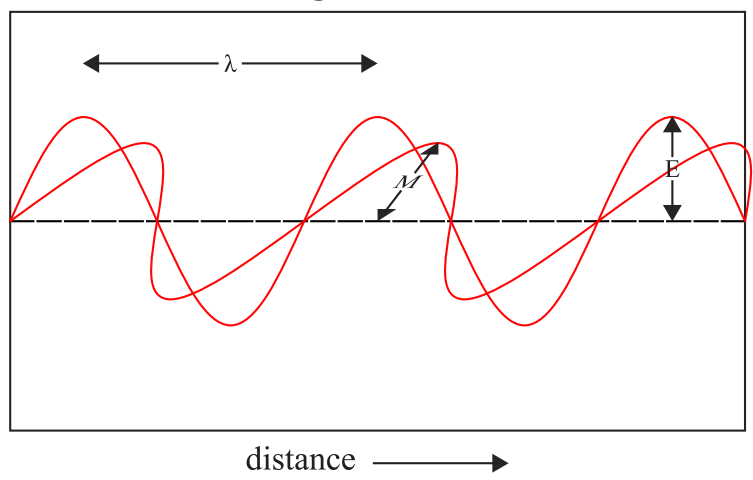

$\lambda=$ wave length

$\mathrm{E}=$ amplitude of electric field

$\mathrm{M}=$ amplitude of magnetic field

Figure B.9. Here, linearly polarized light is illustrated as a transverse wave with electric and magnetic fields oscillating at right angles to the direction of propagation. Image CC BY-SA by Wikimedia user Gpvos.

As discussed above, light can be described as a transverse wave, as shown in Figure B.9. If you look at the figure, you'll see that there are actually two light waves moving in the direction of the arrow: one wave that ripples up and down, and a second wave that ripples left and right. This diagram is more realistic than one might imagine: at the most fundamental quantum level, light from the Sun, a fire, or a hot stove is actually a mixture of two kinds of light: that is, light that is polarized vertically, and light that is polarized horizontally. We can say that this light is disorganized, but it's more common to say that it is not polarized. (This is similar to saying that white light is not colored light, when in fact, white light is actually made up of light of many colors.)

The blue light from the sky on a cloudless day is strongly polarized if you measure it in some directions but not others; it's likely that some birds that fly long distances use this fact to navigate. ${ }^{24}$ Light that reflects off flat water tends to be horizontally polarized, and dragonflies make use of this because their eyes can detect the imbalance. Polarized sunglasses do the same: whereas traditional sunglasses absorb both kinds of polarized light, polarized sunglasses are positioned so that vertically polarized light can pass through while horizontally polarized light is blocked. Such sunglasses do a great

${ }^{24}$ Horváth, Polarization Patterns in Nature: Imaging Polarimetry with Atmospheric Optical and Biological Applications (2003). 


\section{Colaroid's First Product Wasn't a Camera}

Large polarizing films and filters became cheaply available after Edwin Land (1909-1991) invented a way to attach crystals that polarized to film such that all of the crystals lined up. Crystals that polarized light were well known when Land became interested in the topic as an undergraduate at Harvard University: such crystals had been widely used since the 1850s in polarizing kaleidoscopes, entertaining toys which were commonly found in middle-class houses. Scientists wanted to produce large polarizing crystals to make it easier to use polarized light in microscopy and for experiments, but the crystals were fragile and resisted attempts to grow them large. Land's breakthrough discovery in 1928 was that he could grow many tiny crystals and then force them to line up by squeezing a colloidal suspension of the crystals through long narrow slits. ${ }^{a}$ Land left Harvard, perfected the technique, returned to Harvard, then established the LandWheelwright Laboratories in 1932 with his Harvard physics instructor, George Wheelwright, and quit Harvard again. (Land never graduated from Harvard, a fate that would befall other notable entrepreneurs who enrolled as undergraduates but never managed to pull their diploma over the finish line.) The company was renamed the Polaroid Corporation after its primary product in 1937, although it would eventually become better known for its developments in instant photography, electronics, optics, and mechanical engineering.

${ }^{a}$ Robson, "Profile Edwin H. Land" (1984).

job cutting glare from water, roads, and even other cars: they also let people on boats to see better beneath the surface of the water, which is great for fishing.

For outdoor photography, a polarizing filter attached to the front of a camera will preferentially dim the polarized light from the blue sky compared to the clouds, which has the result of intensifying the clouds and producing spectacular photos (see Figure B.10). Years ago these filters were commonly mounted on a rotatable annulus, so that the photographer could turn the filter as appropriate to maximize the intensity of the clouds while turning the sky to a deep blue. These days, it's more common to purchase filters that can create 


\section{Circular Polarizers and 3D Glasses}

Circular polarizers are also responsible for the revolution in $3 \mathrm{D}$ movies. The key behind the illusion of depth in these movies is that each eye is presented with a slightly different view, something called a stereoscopic image. The brain is sensitive to the slight differences between the two images, which creates the illusion of depth. The first 3D movies were black-and-white affairs, with one image projected using red light, the other blue. Viewers wore cardboard glasses with red and blue filters, such that each eye only saw one image. (Red and blue were chosen because they are at opposite ends of the visible light spectrum, which makes it easier to create highly efficient filters that pass one kind of light while blocking the other.) This technique was invented in 1915 and used in comic books and in movies from the 1950s through the 1980s.

Polarized light makes it possible to project 3D movies in color. The early systems used two linear polarizers, typically placed at $45^{\circ}$ and $135^{\circ}$. The problem with these systems was that moviegoers had to sit up straight: any tilt of the head would ruin the $3 \mathrm{D}$ effect. That's why modern $3 \mathrm{D}$ systems use circular polarization: one eye receives light that's polarized in a clockwise direction, the other in a counterclockwise direction. Rather than use two projectors that need to be precisely aligned, it's common to use a single projector with an electrically controlled liquid crystal filter that can rapidly switch polarizations, so that alternating frames go to the left and right eyes.

light that is circularly polarized: it gives the photographer a little less control, but it's easier to use because the photographer doesn't need to worry about orientation. (See the sidebar "Circular Polarizers and 3D Glasses" on page 508 for more information.)

The polarization of light holds an important place in quantum information science because it is the underlying phenomenon on which quantum key distribution, also known as quantum cryptography, is based. It is also one of those quantum effects that are visible at the macroscopic scale and with our human senses.

Here is a simplification of the mathematics of polarization: every photon is polarized in one of two directions, and those directions are determined by how the polarization is measured. So if we are mea- 


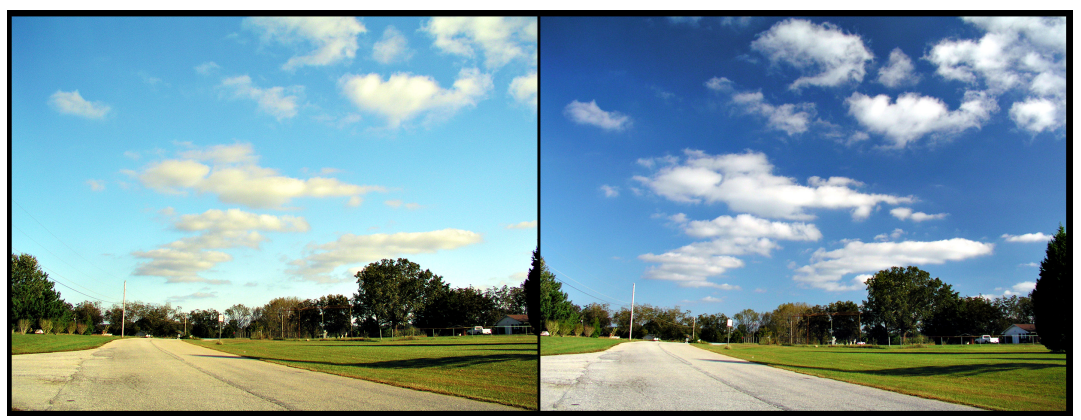

Figure B.10. The effects of a polarizing filter on the sky in a photograph. The picture on the right uses the filter. Image CC BY-SA by Wikimedia user PiccoloNamek.

suring the polarization of light with a linear polarizing filter that is horizontally aligned, the photons that pass through the filter are said to be horizontally polarized while those that do not are vertically polarized. If we are sitting in a 1980s 3D movie, the photons that enter our right eye might be polarized at $45^{\circ}$, while those that go in our left would be polarized at $-45^{\circ}$ or $135^{\circ}$. And if we are in a modern $3 \mathrm{D}$ movie, then the photons that go into the right eye may be circularly polarized in the clockwise direction, while those that go in the left eye may be polarized in the counterclockwise direction. No matter how you measure it, countless scientific experiments have confirmed that there is apparently just one $b i t^{25}$ of polarization state within the photon: the photon can either be aligned with your polarization measurement, or it can be opposed to it. That's because, at the quantum level, polarization is simply the manifestation of something called angular momentum. You can think of circularly polarized photons as tiny spinning corkscrews zipping off at the speed of light in some particular direction.

\section{B.3.1 Six Experiments with Quantum Polarization}

With these concepts of polarization, the next section introduces six experiments that you can do yourself. You will need three linear

${ }^{25} \mathrm{~A}$ bit is a binary digit, colloquially thought of as a 0 or a 1 , or as the values "false" and "true." Claude E. Shannon (1916-2001), the "father" of information theory, attributes the word to the American mathematician John W. Tukey (1915-2000), although the word was in usage before Claude gave it a precise mathematical definition in 1948. See Garfinkel and Grunspan, The Computer Book (2018). Bits are discussed on p. 86. 


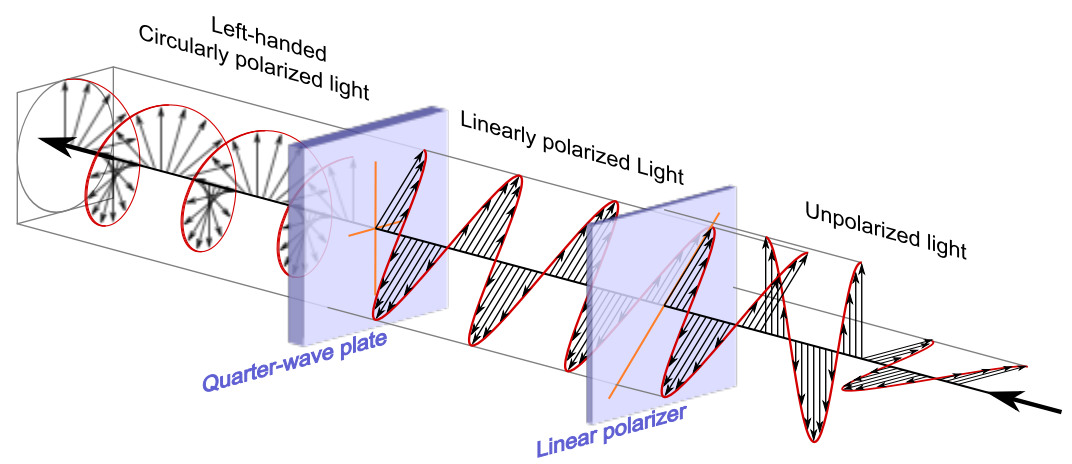

Figure B.11. This illustration shows differences in the waves among disorganized, linearly polarized, and circularly polarized light moving from right to left. Disorganized, or unpolarized, light, characterized by a mixture of polarizations, appears on the right side. The first file manipulated the waves into linearly polarized light (center); the second to circularly polarized light (left). Image public domain by Wikimedia user Dave3457; see Wikipedia for a more detailed explanation.

polarizing filters. (Don't use a circular polarizing filter: you won't get the same results.)

Experiment 1: A Single Linear Polarizing Filter Take a single sheet of a linear polarizing filter and look through it at an incandescent light bulb (if you can find one), a burning candle (be careful not to catch the filter on fire!), or a red-hot stove. All of these objects emit black-body radiation with roughly equal amounts of photons polarized in each direction. (If you don't have any of those, just use a white wall.) You'll see that the filter decreases the intensity roughly by half, but you shouldn't see anything special (Figure B.12, left pane). We will call this the $\leftrightarrow$ direction, or a $0^{\circ}$ rotation.

What's happening here is that light that has linear polarization that's aligned with the filter passes, while light that is not aligned with the polarizer does not pass. If you use a light meter, you'll see that roughly half of the light is blocked.

Experiment 2: Two Linear Polarizing Filters at $\left(0^{\circ}, 0^{\circ}\right)$ Now take two linear polarizing filters (Figure B.12, right pane), hold them at the same angle, and look through both of them together. You'll see that the light passes through, and it's about the same strength as when passing through a single filter. Schematically, this is $\leftrightarrow \leftrightarrow$, or 

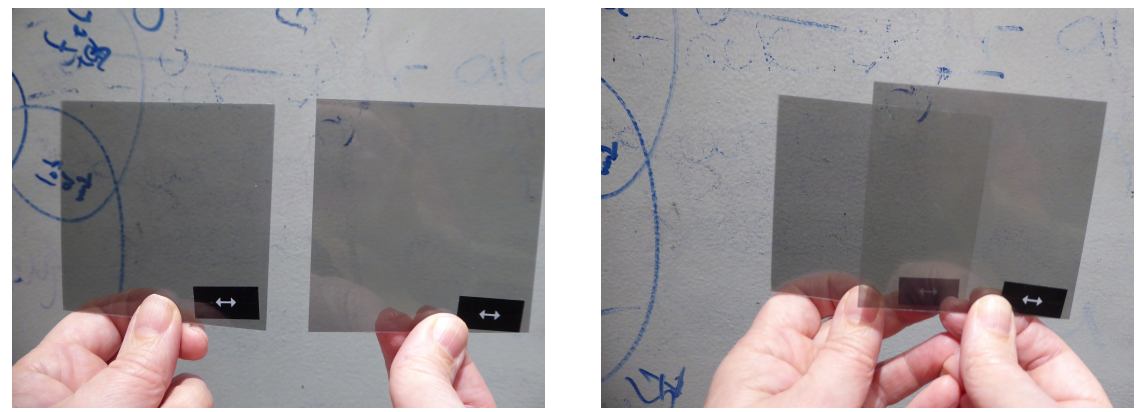

Figure B.12. Two linear polarizing filters with their polarization angles aligned (experiment 1 , left), and overlapping (experiment 2 , right) at $\leftrightarrow$.

two filters at $0^{\circ}$ rotation each. Using the logic introduced in Experiment 1 , the light that makes it through the first filter is polarized in the $\leftrightarrow$ direction, so it can pass through the second filter as well.

Experiment 3: Two Linear Polarizing Filters at $\left(0^{\circ}, 9^{\circ}\right)$ Rotate one of the filters $90^{\circ}$, so that one filter is $\leftrightarrow$ and the other is $\uparrow$ (that is, $0^{\circ}$ and $90^{\circ}$, as in Figure B.13, right pane). Position the filters so that you can look through either $\leftrightarrow$ filter or both the $\leftrightarrow$ and the $\uparrow$ filter at the same time. You'll see that the combination of the two filters blocks nearly all the light. Using the logic introduced in Experiment 1, the light that makes it through the first filter is polarized in the $\leftrightarrow$ direction, and when it arrives at the second filter it can't pass.

Experiment 4: Two Linear Polarizing Filters at $\left(0^{\circ}, 45^{\circ}\right)$ Experiment 3 observed the interaction of light and two filters, one at $0^{\circ}$, one at $90^{\circ}$. If instead the filters are oriented at $0^{\circ}$ and $45^{\circ}$, that is, at $\uparrow$ and $\nwarrow$, there is still no surprise (Figure B.13, left pane). Roughly half of the light ( $50 \%$, or 0.5 ) passes through the first filter, and roughly half of that light passes through the second. So the combination of the $0^{\circ}$ and the $45^{\circ}$ filter lowers the light to $25 \%$ or .25 of its original intensity.

Experiment 5: Three Linear Polarizing Filters at $\left(0^{\circ}, 9^{\circ}\right.$, $\mathbf{4 5}^{\circ}$ ) Now take three filters and arrange them as $\uparrow \leftrightarrow \nwarrow$. You will see the same lack of light passing through the three filters as you saw with the two $\mathfrak{\imath} \leftrightarrow$ filters. There are no surprises here. Only light 

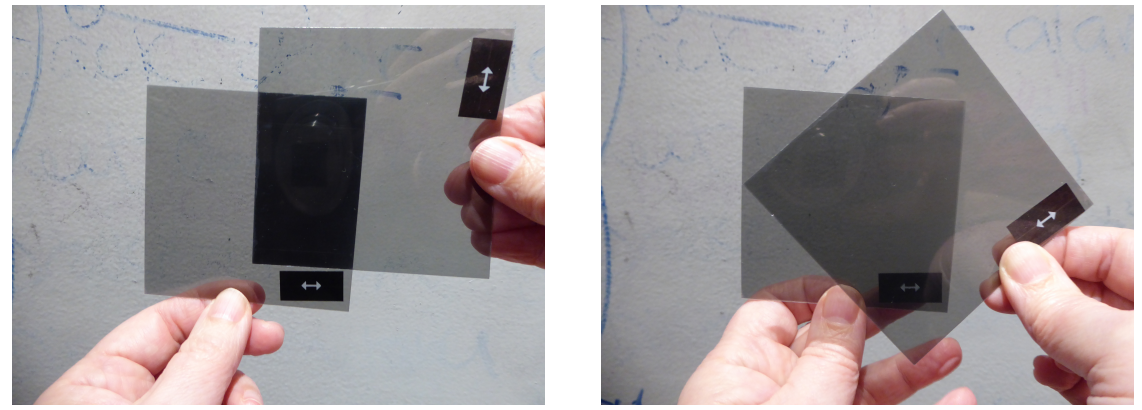

Figure B.13. Two overlapping linear polarizing filters with a $90^{\circ}$ angle between their polarization angles (experiment 3 , left) and a $45^{\circ}$ angle (experiment 4 , right). Notice that at $90^{\circ}$, no light comes through, whereas at $45^{\circ}$ roughly half of the light comes through.
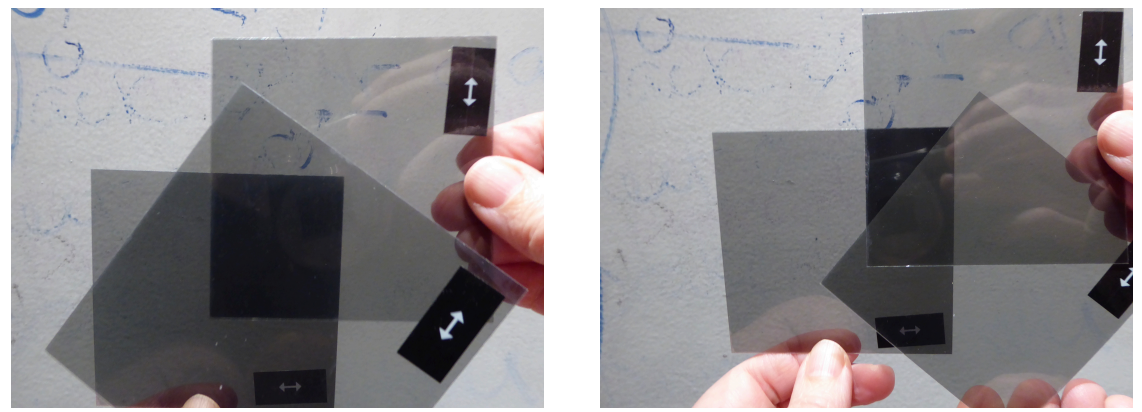

Figure B.14. Three overlapping linear polarizing filters in two different orientations. In both cases the rearmost filter is at $0^{\circ}$. On the left (experiment 5 ) the middle filter is at $90^{\circ}$, and the one closest to the camera is at $45^{\circ}$. Notice that the filter at $0^{\circ}$ combined with the filter at $90^{\circ}$ blocks all of the light; the filter at $45^{\circ}$ has no effect. On the right (experiment 6 ), the middle filter is at $45^{\circ}$, and the one closest to the camera is at $90^{\circ}$. Notice that the triangle showing where the $0^{\circ}$ and $90^{\circ}$ filter overlap is actually darker than the four-sided shape in the middle where the filters are stacked at $0^{\circ}$, then $45^{\circ}$, then $90^{\circ}$.

that is polarized in the up-down direction passes through the first filter. That light can't pass through the second filter. The third filter is present, but it doesn't do anything. See Figure B.14.

Experiment 6: Three Linear Polarizing Filters at $\left(0^{\circ}, 45^{\circ}\right.$, $\mathbf{9 0}^{\circ}$ ) Given the results of Experiment 5, what happens if one reverses the order in which light passes through the $90^{\circ}$ and the $45^{\circ}$ 
filter? That is, what happens if the light encounters the filters as a stack of $\uparrow \nwarrow \leftrightarrow\left(0^{\circ}, 45^{\circ}, 90^{\circ}\right)$ ? See Figure B.14 right pane.

Before answering the question about experiment 6 , you'll note that what was happening in Experiment 4, when the light that passed through the $0^{\circ}$ filter suddenly encountered the $45^{\circ}$ filter, went unexplained. Why would roughly half of the light make it through, and is it roughly half, or is it exactly half?

Polarization can be thought of as the direction of oscillation of the transverse wave, or as the angular momentum (or spin) of each photon. So the light that passes through the first filter is oriented at $0^{\circ}(\uparrow)$. When this light hits the filter oriented at $45^{\circ}$, it has a $50 \%$ chance of passing through and a $50 \%$ chance of being absorbed ${ }^{26}$ But now the light passing through the second filter has a polarization of $45^{\circ}$, so when this light hits the third filter, there is once again a $50 \%$ chance that the light will pass through and a $50 \%$ chance of it being absorbed. As a result, when the filters are at $0^{\circ}, 45^{\circ}$, and $90^{\circ}$, the amount of light passing through the first filter is $50 \%$, the amount of light passing through the second is $25 \%$, and the amount of light passing through the third is $12.5 \%$ of the original.

Once the photon passes through the first $0^{\circ}$ filter, it is absolutely certain that it will pass through a second $0^{\circ}$ filter and be blocked by a $90^{\circ}$ filter. But if the photon encounters a $45^{\circ}$ filter before the $90^{\circ}$ filter, then all bets are off: the photon might pass, or it might be blocked by the $45^{\circ}$, and if it passes through, then it might be blocked by the $90^{\circ}$, or it might pass through. This is a direct result of the photon only having a single bit of internal state to represent the direction of its angular momentum: it's either polarized horizontally or vertically, it's polarized at $45^{\circ}$ or $-45^{\circ}$, or it's spinning clockwise or counterclockwise. One set of measurements gives no information about the other set of measurements.

\section{B.4 Quantum Effects 3: Entanglement}

This section turns to the phenomenon known as quantum entanglement.

Entangled particles are particles that are somehow linked on the quantum level, even though they are physically separated with no

${ }^{26}$ The amount of light passing through can actually be calculated using the Born Rule as $\cos (\theta)^{2}$ where $\theta$ is the angle between the polarization of the first filter and the second filter. Note that $\cos (45 \mathrm{deg})^{2}=.5$. 
way to communicate. Entanglement has no direct analog in the classical world, and it is so strange that Einstein labeled it "spooky actions at a distance." ${ }^{27}$ One way to think of it is that entangled particles are part of a system, where measuring any part of the system reveals information about other parts.

When particles are entangled, measurement of one causes the other to act in a predictable fashion. Entanglement appears to violate relativity, because measurement appears to cause the other particle to react instantly, superluminally, even when the particles are separated by great distances. Spooky action occurs without sending information through physical space. Sometimes it is said that entanglement enables communication at faster-than-light speeds, but this is impossible, as discussed in the sidebar "Alas, Faster-than-light Communication Is Not Possible" on page 301.

One of the simplest systems of entangled particles is a pair of photons released when a high-speed laser pulse strikes a special kind of crystal that has been pumped into a high-energy state. Spontaneous parametric down conversion (SPDC) is a common method to create entangled photons. In SPDC, the laser pulse, striking a beta barium borate crystal, causes the crystal to release two complementary photons traveling in opposite directions. Because they are created together, angular momentum is conserved, which means that if one photon is spinning clockwise as it zips through space, then the other must be spinning counterclockwise. So far, so good.

Recall that each photon's angular momentum is related to how it will interact with a linear polarizing filter. If one photon will pass through a polarizing filter that's oriented at $0^{\circ}$, then the other one will pass through a filter oriented at $90^{\circ}$. If one photon passes through a filter oriented at $45^{\circ}$, the other one will pass through a filter oriented at $-45^{\circ}$. So if one sets up two filters, one for each photon, and the filters are oriented at $0^{\circ}$ and $90^{\circ}$, then the entangled photons will either pass through both of the filters, or they will pass through neither of the filters. On the other hand, if both of the filters are oriented $0^{\circ}$, then one of the entangled photons will pass through one of the filters and the other entangled photon will be absorbed.

What happens if instead of placing the filters at $0^{\circ}$ and $90^{\circ}$, the two filters are placed at $0^{\circ}$ and $45^{\circ}$ ? Unlike polarization at $0^{\circ}$ and

${ }^{27}$ Einstein and Born, The Born-Einstein Letters: Correspondence between Albert Einstein and Max and Hedwig Born From 1916-1955, with Commentaries by Max Born (1971). 
$90^{\circ}$, or clockwise and counterclockwise, polarization at $0^{\circ}$ and $45^{\circ}$ are unrelated at the quantum level. Just as half of the light that passed through the $0^{\circ}$ filter could pass through a $45^{\circ}$ filter, if the first photon encounters a $0^{\circ}$ filter and the second encounters a $45^{\circ}$ filter, then each photon will have a $50 \%$ chance of passing through to the other side: there will be no correlation between the two measurements.

This is the essence of entanglement: it is also the essence of quantum key distribution. And it was profoundly disturbing to Albert Einstein, Boris Podolsky, and Nathan Rosen, who identified the problem when they were working together at the Institute for Advanced Study in Princeton, NJ in 1934, and published their classic paper on the topic in 1935: "Can Quantum-Mechanical Description of Physical Reality Be Considered Complete?" - known as the EPR paper.

Simply put, here is the paradox that the EPR paper identifies: because they are moving in opposite directions at the speed of light, there is no way for the two photons to communicate with each other. Nothing, after all, can move faster than the speed of light. If the first photon hits a polarizing filter at $0^{\circ}$ and the second hits a filter at $90^{\circ}$, only one of them will pass through. But if they hit filters at $0^{\circ}$ and $45^{\circ}$, then each photon has a $50 \%$ chance of passing through. Run a lot of experiments in which two entangled photons hit a pair of filters at $0^{\circ}$ and $45^{\circ}$, and roughly a quarter of the time neither photon will pass, a quarter of the time the A photon will pass, a quarter of the time the B photon will pass, and a quarter of the time both photons will pass. This happens even if the orientation of the polarizing filters is set after the entangled photons are created.

How does each photon know the orientation of both filters at the time of impact?

The challenge here is that the two entangled photons are described by a single wave function. This made no sense to the scientists. What holds the photons together? If the photons were in some kind of communication, it would need to be faster than the speed of light, and that would violate Relativity. On the other hand, by 1934 wave mechanics was well enough developed that it had accurately predicted the outcome of every experiment designed to test it: wave mechanics was clearly correct. Therefore, the EPR paper argued, the description of reality provided by quantum mechanics must not be complete - there must be more to the description of each entangled photon than its wave function. Or, as the paper states it: 
From this follows that either (1) the quantum-mechanical description of reality given by the wave function is not complete or (2) when the operators corresponding to two physical quantities do not commute the two quantities cannot have simultaneous reality. For if both of them had simultaneous reality - and thus definite values - these values would enter into the complete description, according to the condition of completeness. If then the wave function provided such a complete description of reality, it would contain these values; these would then be predictable. This not being the case, we are left with the alternatives stated. ${ }^{28}$ (emphasis in original)

In his March 1947 letter to Born, Einstein put his objection into more colorful language:

I cannot make a case for my attitude in physics which you would consider at all reasonable. I admit, of course, that there is a considerable amount of validity in the statistical approach which you were the first to recognise clearly as necessary given the framework of the existing formalism. I cannot seriously believe in it because the theory cannot be reconciled with the idea that physics should represent a reality in time and space, free from spooky actions at a distance. $^{29}$

Entanglement is a powerful technique that is central to quantum computing, metrology (the study of measurement), sensing, and communication. In quantum computing, entanglement is used to create coordinated ensembles of particles. Operating together, these ensembles may provide faster computing in a quantum computer. In metrology and sensing, an entangled photon can illuminate an object while the linked particle can be measured to learn about the target. In communication, entanglement can be used to create random sequences of bits that can be used as encryption keys for securely exchanging information even in the presence of surveillance. As will be seen in

${ }^{28}$ Einstein, Podolsky, and Rosen, "Can Quantum-Mechanical Description of Physical Reality Be Considered Complete?" (1935).

${ }^{29}$ Einstein and Born, The Born-Einstein Letters: Correspondence between Albert Einstein and Max and Hedwig Born From 1916-1955, with Commentaries by Max Born (1971), p. 158. 
Chapter 7, in 2017, Chinese researchers maintained entangled photons at $1200 \mathrm{~km}$ using a satellite that communicated with two base stations. As Science explained it, "Spooky action achieved at record distance." 30

The EPR paper argues that there must be some deeper theory from which the probabilistic quantum theory could be derived. That theory would presumably assign to particles like photons and electrons additional state that would be described by new variables, and from those variables the observed probabilities could be derived. From the point of view of this underlying, more complete, and utterly hypothetical theory, there would be no randomness. Today this is called the "hidden variable theory."

In 1964 physicist John Stewart Bell developed a hypothesis that would need to be true for any explanation of quantum mechanical results based on hidden variables. ${ }^{31}$ In the years that followed, experiments were designed that could prove or disprove the hypothesis: these were called Bell tests. In the intervening years, these experiments have been carried out with ever-increasing precision and levels of exactness. The conclusion of this line of work is now clear: entanglement exists. Entangled particles are somehow linked. There are no hidden variables.

\section{B.5 Quantum Effects 4: Superposition}

Let us go back to our experiments with light and linear polarizing filters. Recall that if a photon passes through the first filter at $0^{\circ}$, it will pass through a second filter at $0^{\circ}$, but it only has a $50 \%$ chance of passing through a filter at $45^{\circ}$, and it has a $0 \%$ chance of passing through a filter at $90^{\circ}$.

One of the reasons that Schrödinger's wave equation (described more fully below) was such a breakthrough is that it gave physicists a mathematical approach for describing this situation. Once the equation is written down it's then possible to solve for the amount of light that passes through the second filter. If $p$ is the fraction of light that passes through the second filter after passing through the first, and $\theta$ is the angle between the two filters, then the equation is $p=\cos ^{2}(\theta)$, where cos is the trigonometric cosine function that evaluates to 0 at $0^{\circ}, 1$ at $90^{\circ}$, and $\sqrt{0.5}$ at $45^{\circ}$. The function is squared in line with Born's rule.

\footnotetext{
${ }^{30}$ Popkin, "Spooky Action Achieved at Record Distance" (2017).

${ }^{31}$ Bell, "On The Einstein Podolsky Rosen Paradox" (1964).
} 


\section{-Man Plays Dice with Einstein's Words}

Einstein never said, or wrote, one of the most famous quotations attributed to him - that God does not play dice with the Universe. In his December 4, 1926 letter to Max Born, Einstein actually wrote:

"Die Quantenmechanik ist sehr achtunggebietend. Aber eine innere Stimme sagt mir, daß das noch nicht der wahre Jakob ist. Die Theorie liefert viel, aber dem Geheimnis des Alten bringt sie uns kaum näher. Jedenfalls bin ich überzeugt, daß der nicht würfelt." $a$

The Hebrew University of Jerusalem, Israel owns the copyright on the letter. In the 2005 publication of Einstein and Born's letters, the German was thus translated:

"Quantum mechanics is certainly imposing. But an inner voice tells me that it is not yet the real thing. The theory says a lot, but does not really bring us any closer to the secret of the "Old One." I, at any rate, am convinced that He is not playing at dice."

Here's another translation, with commentary:

"Even 'God does not play dice,' arguably Einstein's most famous quote, isn't quite his words. It derives from a letter written in German in December 1926 to his friend and sparring partner, theoretical physicist Max Born. It is published in the new volume of Einstein's papers, in which the editors comment on its 'varying translations' since the 1920s. Theirs is: 'Quantum mechanics ... delivers much, but does not really bring us any closer to the secret of the Old One. I, at any rate, am convinced that He does not play dice.' Einstein does not use the word 'God' (Gott) here, but 'the Old One' (Der Alte). This signifies a "personification of nature," notes physicist and Nobel laureate Leon Lederman (author of The God Particle, 1993). ${ }^{c}$

${ }^{a}$ Einstein, Born, and Heisenberg, Albert Einstein Max Born, Briefwechsel 1916-1955: Mit Einem Geleitwort von Bertrand Russell (Deutsch) (2005).

${ }^{b}$ Einstein and Born, The Born-Einstein Letters 1916-1955: Friendship, Politics and Physics in Uncertain Times (2005).

${ }^{c}$ Robinson, "Did Einstein Really Say That?" (2018). 
If you are wondering why $p$ was used in the above paragraph for the fraction of light passing through, rather than $f$, that's because the equation really isn't about the fraction of light passing through: it provides the probability that any particular photon that passes through the first filter will pass through the second. This probability (which ignores the probability that the photon will be absorbed by the substrate on which the polarizing material rests) holds true in general for any pair of polarizing filters.

- This is why $12.5 \%$ of the light that enters a sandwich of three polarizing filters at $0^{\circ}, 45^{\circ}$, and $90^{\circ}$ will pass through: $50 \%$ will pass through the first filter at $0^{\circ}, 50 \%$ of that light will pass through the filter at $45^{\circ}$, and then $50 \%$ of that light will pass through the filter at $90^{\circ}$.

- This is also why $0 \%$ of light will pass through a sandwich of filters at $0^{\circ}, 90^{\circ}$, and $45^{\circ}: 50 \%$ of the light will pass through the first filter at $0^{\circ}$, then $0 \%$ will pass through the filter at $90^{\circ}$. And that's that. There's no more light. If there was light leaving the filter at $90^{\circ}, 50 \%$ of it would pass through the filter at $45^{\circ}$. But there isn't any light, so nothing passes through.

The word superposition can be used to describe what's happening here at the quantum level. In quantum mechanics, the Schrödinger wave equation allows any wave to be described as a combination ${ }^{32}$ of any other waves. Physicists and engineers can use this property to describe physical systems with simplified wave equations that focus on the particular quantum phenomena on which they are focusing, or they can write exceedingly complex wave equations with many terms to consider more possibilities (or simply to impress their friends and intimidate their rivals).

To get a better understanding of what might be happening in the case of the three polarized filters, each photon approaching a polarizing filter can be described as a superposition of two photon possibilities: the possibility that the photon will travel through the filter, and the possibility that the photon will be absorbed. If these are the only two possible outcomes - that is, if one ignores the possi-

\footnotetext{
${ }^{32}$ In quantum mechanics, the waves are actually represented as linear functions of other waves, which means that waves can be added or subtracted in any proportion, but cannot be multiplied or divided.
} 


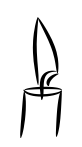

White Light

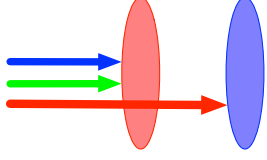

Red

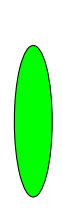

Green
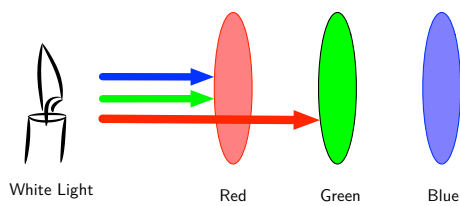

Figure B.15. Photons in the white light from the candle are represented here as colored lines containing photons from the red, green, and blue regions of the visible spectrum. These photons are all at different energy levels, with the blue photons having roughly twice as much energy per-photon as the red photons. When the white light encounters the red filter, only the red photons can pass through: the rest are absorbed, which is why the world looks red when you look through the filter. The red photons, in turn, are blocked by the blue filter (left) or the green filter (right). Thus, no light can pass through any combination of red, green, and blue filters, no matter which order the light encounters the filters. (Illustration credit: Simson Garfinkel)

bility that the photon might be reflected off the surface of the filter - then these two possibilities must sum to 1 :

$$
1=p_{\text {pass }}+p_{\text {absorb }}
$$

Recall that the probability of a the photon passing through was $\cos ^{2}(\theta)$. So another way of writing this equation is:

$$
1=\cos ^{2}(\theta)+p_{\text {absorb }}
$$

which is equal to:

$$
1=\cos ^{2}(\theta)+\sin ^{2}(\theta)
$$

From a wave mechanics point of view, this is actually a summation of two wave equations: one that represents the probability that the photon will pass through the filter, and the other representing the probability that the photon will be absorbed.

The remainder of this section will discuss why behavior of the three polarizing filters at $0^{\circ}, 45^{\circ}$, and $90^{\circ}$ seems so strange, by explaining what's happening at the quantum level if colored filters were used instead of polarizing ones.

Most of us have a clear understanding of how light passes through colored glass as a result of our day-to-day experiences and from color theory. White is made up of all the colors of the rainbow. Red light passes through red glass and blue light passes through blue glass (Figure B.15). This is why blue things look black through a red 
filter, and red things look black through a blue filter. Old-style 3D movies and comic books were based on this basic optics.

Polarized light doesn't work this way. Although it's tempting to think that polarizing filters act like colored gels, except that they let through light that is aligned as little arrows $\left(\leftrightarrow\right.$ for a filter at $0^{\circ}, \uparrow$ for a filter at $90^{\circ}$ ), that's not what is happening. If it was, then only a tiny bit of light could possibly make it through a polarizing filter set at $0^{\circ}$ - not only would the light at $90^{\circ}$ be absorbed, but so too would the light at $45^{\circ}$ be blocked.

Color and polarization are different, because individual photons really do have individual color - a photon's color is directly related to its wavelength, which is a real thing that you can measure in many different ways. A photon's polarization, in contrast, is a superposition of wave functions. Those wave functions are determined by the photon's angular momentum, or spin.

Candles emit a stream of photons in every direction. Any individual photon's spin is going to be in one direction or the other, but overall the numbers will be equal because angular momentum is conserved. So when one of these photons hits that first polarizing filter, it has a $50 \%$ chance of traveling through, and a $50 \%$ chance of being absorbed.

If that photon travels through the filter, its polarization is now aligned with the crystals out of which the filter was built. When that photon comes to a filter that's $45^{\circ}$ out of alignment, there is only a $50 \%$ chance that the photon will properly interact with the crystals in the second filter and pass through. But if it does, its polarization is now aligned with the second set of crystals. If you want a classical, non-wave-equation way of thinking about this, you can pretend that the second filter turned the photons that successfully passed through (Figure B.16). If you want a quantum mechanical explanation, you could say that the wave function describing photons on the left side of the $45^{\circ}$ filter describes a superposition of photons that can pass through the filter and those that cannot; likewise the wave function that describes photons on the right side of the filter is a superposition of those that did pass through the filter and ... well, and nothing. But that wave function can itself be described as a superposition of photons that can pass through a filter at $0^{\circ}$ and those that can pass through a filter at $90^{\circ}$. 

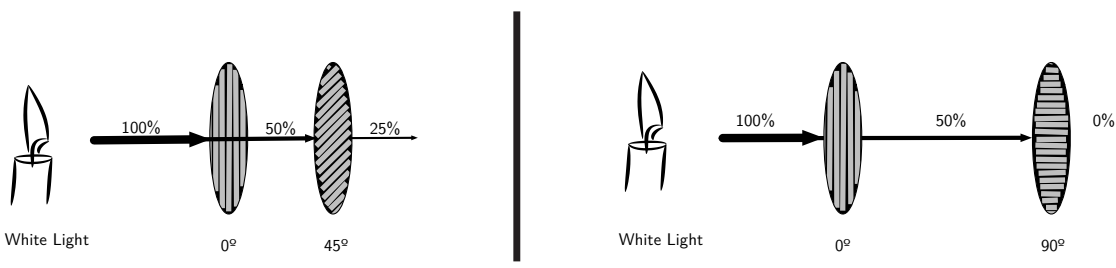

Figure B.16. Photons in the white light from the candle are represented here with black lines that represent the stream of photons leaving the candle. Each photon contains two possible polarizations (or two possible angular momenta). When this stream of photons hits a linear polarizing filter at $0^{\circ}$, only $50 \%$ of the photons can pass through. These photons have now been measured to have a linear polarization of $0^{\circ}$. If these photons interact with a linear polarizing filter that has a $45^{\circ}$ offset (left), $50 \%$ of the photons can pass, because $\cos ^{2}(45 \mathrm{deg})=0.5$. Alternatively, if these photons interact with a linear polarizing filter that has a $90^{\circ}$ offset (right), none of the photons can pass, because $\cos ^{2}(90 \mathrm{deg})=0$.
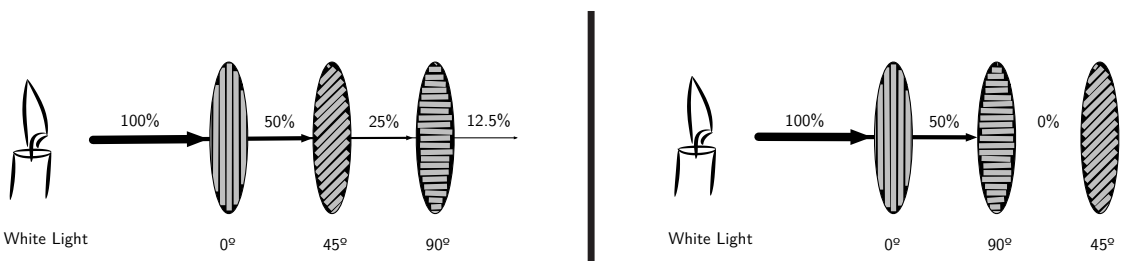

Figure B.17. In this example, light is directed to travel through three polarizing filters. On the left, the light passes through the filters that are set at the angles $0^{\circ}, 45^{\circ}, 90^{\circ}$, which means that the photons encounter two transitions of $45^{\circ}$, one after the other. Each transition reduces the amount of light that passes by $50 \%$. On the right the light is set to pass through the filters that are set at the angles $0^{\circ}, 90^{\circ}, 45^{\circ}$, which means that the photons should first encounter a transition of $90^{\circ}$ and then one of $-45^{\circ}$. However the first transition blocks all of the light.

\section{B.6 The Cat State}

The experiments that we've presented in this appendix and the explanations for the somewhat paradoxical results are much simpler (and correspondingly less accurate) than you are likely to find in other books on quantum information science, let alone on quantum mechanics. Although many quantum devices are based on polarized light, they don't measure whether photons are transmitted or absorbed: instead, they send the photon into a crystal that either reflects or transmits the light depending on its phase, and then they use two sensors, each capable of detecting a single photon.

Complex two and four-beam systems are not discussed in this appendix because simplifying the presentation enables one to demonstrate with experiments using three low-cost and mass-produced lin- 
ear polarizing filters, while reading this very book. This approach can give one an intuitive feel for the strangeness of quantum mechanics - a strangeness that arises because the behavior of tiny quantum particles is so very different than the behaviors we observe at the macroscopic scale.

This book also intentionally avoids any discussion of what the Schrödinger wave equation actually means - whether there is a wave function that collapses when it is measured or observed, as the socalled Copenhagen Interpretation of quantum mechanics holds, or whether the universe literally splits in two, as is held by the alternative many-worlds interpretation. And no time is devoted here to the vastly less popular pilot wave theory, first proposed by de Broglie, then rediscovered by David Bohm in 1952, which holds that the probabilistic interactions are themselves an illusion, and that the wave function describes a real wave that pushes around real particles. In pilot wave theory, the pilot wave is the wave described by the Schrödinger Wave Equation; a second equation called the Guiding Equation describes how the wave moves. Pilot wave theory does require hidden variables, but they are global: the entire wave function is instantaneously affected by every other particle in the universe.

Schrödinger and Einstein were both fundamentally dissatisfied with a theory of reality that depended so intimately upon the role of the observer. To that end, they created a thought experiment that today is referred to as Schrödinger's cat. The idea was to imprison a cat and a radioactive source in a box that has no contact with the outside world. There is a $50 \%$ chance that the radioactive material will decay within an hour and that the decay will be detected by a machine that's also in the box. If the decay is detected, the machine kills the cat - either by releasing poison gas (in Schrödinger's version) or by blowing up some explosive (Einstein). So at the end of the hour, the cat is either alive or it's dead. But since quantum mechanical events aren't actually settled until they are observed (at least, that's the story according to the Copenhagen interpretation), then the cat is both alive and dead until someone opens the box and checks on it. Unless the cat is also an observer, in which case it either observes that it's alive, or it's dead.

Perhaps the cat is both alive and dead: perhaps the universe has split in two, and there are really two cats. That's the many-worlds interpretation of quantum mechanics. Or perhaps there is a pilot wave, determined by all of the particles in the universe, and the 
radioactive material either did decay or it didn't, and it was either detected or it wasn't, and the cat is either alive or it's dead, no matter if there is an observer or not. That's the pilot wave theory at work.

In quantum theory, some scientists use the cheeky term "cat state" to describe an object that simultaneously exists in two opposing states. Humanity currently lacks the scientific tools to test these multiple competing interpretations of reality, and because all of them are equally compatible with the quantum devices that are being created in labs today and likely to be created for the foreseeable future, you, dear reader, can choose the ultimate nature of your own physical reality. 